\title{
High-Resolution and Tandem Mass Spectrometry - the Indispensable Tools of the XXI Century
}

\author{
Yury O. Tsybin*, Luca Fornelli, Anton N. Kozhinov, Aleksey Vorobyev, and Sasa M. Miladinovic
}

\begin{abstract}
Mass spectrometry-based qualitative and quantitative (bio)molecular analysis is a corner stone in the state-of-the-art pipelines in systems biology and environmental sciences. High-resolution and efficient tandem mass spectrometry methods and techniques are the essential analytical capabilities for the in-depth analysis of extremely complex mixtures of (bio)molecules of a very broad dynamic range of concentrations. Here, we briefly review the advantages and limitations of the current mass spectrometry with a focus on resolution, or resolving power, and methods of (bio)molecular fragmentation in the gas phase. We conclude with an outlook that considers possible avenues for further mass spectrometry-based method and technique development, indispensable for advancing the challenging real-life mass spectrometry applications in the XXI century.
\end{abstract}

Keywords: Fourier transform ion cyclotron resonance, FT-ICR · Liquid chromatography .

Mass spectrometry, MS · Peptide · Petroleomics · Protein · Proteomics · Tandem mass spectrometry, MS/MS

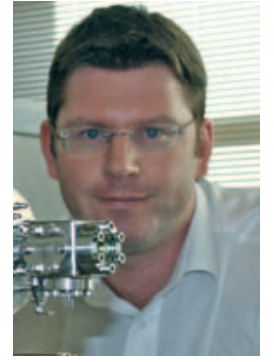

Health, energy and sustainability are the grand challenges the world faces in the XXI century. Recent progress in science and technology now allows these prioritized directions of humanity development to be considered at the molecular level. ${ }^{[1-5]}$ Indeed, understanding fundamental chemical and biological processes in nature and technology today requires knowledge of the identity, quantity, structure and interaction partners of the participating molecules. The near future will rely even more on molecular level science and technology. Mass spectrometry (MS) is a key analytical technique in receiving the required molecular information from minute amounts of samples present in the gas, solid or liquid phase. ${ }^{[2]}$ Molecular information on organic, inorganic and biological molecules can be deduced from the accurately measured masses of the molecules of interest and their fragments. ${ }^{[1,6]}$ The ground-breaking

${ }^{*}$ Correspondence: Prof. Dr. Y. O. Tsybin Biomolecular Mass Spectrometry Laboratory Ecole Polytechnique Fédérale de Lausanne EPFL LSMB BCH 4307 $\mathrm{CH}-1015$ Lausanne

Tel.: +41216939751

Fax: +412169398 85

E-mail: yury.tsybin@epfl.ch developments of soft ionization methods in biological MS in the 1980s, namely electrospray ionization (ESI) ${ }^{[7]}$ and matrix-assisted laser desorption ionization (MALDI), ${ }^{[8]}$ have tremendously accelerated the development of analytical instrumentation and data analysis tools for species of biological importance, leading to the recognition of ESI and MALDI developments with a Nobel Prize in Chemistry in 2002. Particularly, significant investments have been made in the area of the MS-based life sciences applications, especially systems biology-oriented ones. ${ }^{[9,10]}$ The important building platform for this yet emerging field is the '-omics' cascade, including genomics, transcriptomics, proteomics, ${ }^{[1,11,12]}$ and metabolomics. ${ }^{[3]}$ Mass spectrometry-based proteomics and metabolomics heavily rely on high-resolution mass spectrometry and tandem mass spectrometry (MS/MS),[13] briefly reviewed below.

Resolving power is the key analytical characteristic of a mass spectrometer responsible for detecting the molecular signal related to a unique molecule present in the sample and not to the mixture of two or more molecules. ${ }^{[14,15]}$ Sufficient resolving power is thus required not only for the accurate mass assignment to the molecule, but also to achieve required levels of sensitivity and dynamic range to enable the molecule's unambiguous identification and characterization. ${ }^{[16]}$ The required level of resolving power in a typical mass spectrometric experiment is thus a function of sample complexity. The resolving power of a mass spectrometer to a large extent depends on the type of a mass ana- lyzer employed. Mass analyzers separate ions according to their mass/charge $(\mathrm{m} / \mathrm{z})$ ratio. Together with an ion source and an ion detector, the mass analyzer constitutes the three main building blocks of a mass spectrometer. The most widely used mass analyzers for (bio)molecular structure analysis today are time-of-flight and ion trap ones. In general, mass analyzers may be classified by the forces that govern the ion motion in a collision-free or collision-reduced environment (vacuum) and produced by electric and magnetic fields (Table 1). Following this classification, the mass analyzers may be divided into field-free-based mass analyzers (measurement of ion time-of-flight (TOF) in a field-free region); electric field-based mass analyzers (ion resonance filtering or trapping on specific trajectories with electrostatic sector, quadrupole, quadrupole traps, and an Orbitrap); and magnetic field-based mass analyzers (magnetic sector and an ion cyclotron resonance, ICR). Interestingly, there is no mass analyzer that employs both electric and magnetic fields for ion separation by their $\mathrm{m} / \mathrm{z}$ ratio. Nevertheless, electric fields are employed for ion acceleration prior to ion separation, for example by TOF or ICR. In addition, TOF mass analyzers require supplemental ion manipulation to increase the resolving power, e.g. single or multiple ion mirrors (reflectrons) and orthogonal ion extraction devices. ${ }^{[17]}$ Similarly, ICR MS requires an electric field to trap the ions in the direction parallel to the magnetic field. [18]

High magnetic field Fourier transform ion cyclotron resonance mass spectrometry (FT-ICR MS) provides an unbeatable 
Table 1. Classification of modern mass analyzers by the employed electric and magnetic fields.

\begin{tabular}{|c|c|c|c|}
\hline $\begin{array}{l}\text { General } \\
\text { equation of } \\
\text { ion motion }\end{array}$ & \multicolumn{3}{|c|}{$M \frac{d \vec{V}(\vec{r}, t)}{d t}=q \vec{E}(\vec{r}, t)+q[\dot{V}(\vec{r}, t) \times \vec{B}(\vec{r})]$} \\
\hline $\begin{array}{l}\text { Particular } \\
\text { case }\end{array}$ & $\begin{array}{l}\vec{B}(\vec{r})=0 \\
\vec{E}(\vec{r}, t)=0\end{array}$ & $\vec{B}(\vec{r})=0$ & $\vec{E}(\vec{r}, t)=0$ \\
\hline $\begin{array}{l}\text { Particular } \\
\text { equation of } \\
\text { ion motion }\end{array}$ & $M \frac{d \vec{V}(\dot{r}, t)}{d t}=0$ & $M \frac{d \bar{V}(\bar{r}, t)}{d t}-q \dot{F}(\vec{r}, t)$ & $M \frac{d \dot{d}(t, t)}{d t}=u[\vec{V}(\vec{r}, t) \times \bar{B}(\bar{r})-$ \\
\hline \multirow{4}{*}{$\begin{array}{l}\text { Corresponding } \\
\text { mass analyzers }\end{array}$} & \multirow{4}{*}{$\begin{array}{l}\text { Time-of-flight } \\
\text { (TOF) }\end{array}$} & $\begin{array}{l}\text { Electrostatic } \\
\text { sector }\end{array}$ & Magnetic sector \\
\hline & & Quadrupole & Ion cyclotron \\
\hline & & Quadrupole trap & resonance (ICR) \\
\hline & & Orbitrap & \\
\hline
\end{tabular}

analytical performance in terms of highresolution measurements. ${ }^{[19]}$ In the 1980s, the laboratory of Prof. Dr. Tino Gäumann at the EPFL's Institute of Chemical Sciences and Engineering was one of the leading FT-ICR MS laboratories in the world (Fig. 1, left). ${ }^{[20]}$ Following the development of the soft ionization techniques and recognition of the importance of high-resolution MS in the life sciences in the early XXI century, the Biomolecular Mass Spectrometry Laboratory, headed by Prof. Dr. Yury Tsybin, was created and built around the state-of-the-art high magnetic field FT-ICR MS at ISIC (Fig. 1 , right). Why is 'high magnetic field' important? The advantages of high magnetic field FT-ICR MS include: i) resolution, sensitivity, and scan speed scale linearly with magnetic field strength; ii) upper mass limit, maximum trapping time, maximum ion energy and maximum number of trapped ions vary with the square of the magnetic field strength; iii) peak coalescence due to space charge effects and space-charge-related peak distortion varies inversely as the square of the magnetic field strength. The combined effect of the above-mentioned parameters is responsible for setting the lower and upper limit of the achievable dynamic range, as well as for the improvement in mass accuracy. Other areas of platform improvement to advance the field are within proper ion manipulation, including ion formation, transfer, capture, storage, fragmentation, excitation and detection. Advanced and novel signal processing methods have received particular attention in the recent years and will be briefly mentioned below.

Comprehensive structural analysis of (bio)molecules requires information not only on the accurate mass of the intact molecule, but also on the structure-specific fragments of this molecule. ${ }^{[21]}$ Tandem mass spectrometry (MS/MS) refers to the gas-phase fragmentation of (bio)mole- cules, e.g. peptides and proteins, and mass measurements of both the precursor, or parent, ions and the fragment, or daughter, ions. Ideally, tandem mass spectrometry should provide complete information not only on the sequence of amino acids (protein primary structure) but also on the character and localization of post-translational modifications, e.g. phosphorylation and glycosylation. Peptide quantification is another application area of tandem mass spectrometry. The most widely used methods of peptide and protein fragmentation and fragment ion nomenclature are summarized in Fig. 2. The available arsenal of MS/MS techniques allows fragmenting the peptide at all three backbone bonds, i.e. $\mathrm{C}_{\alpha}-\mathrm{C}, \mathrm{C}-\mathrm{N}$ and $\mathrm{N}-\mathrm{C}_{\alpha}$, to yield ions of $a, b, c$ types (containing the $\mathrm{N}$-terminus) and the corresponding $x, y, z$ types (containing the $\mathrm{C}$-terminus). The number of amino acid residues that belong to a given fragment is shown in a subscript, e.g. $b_{2}$ ion contains two amino acid residues, whereas its corresponding $y_{n-2}$ ion would contain (n-2) amino acids, where $n$ is a number of amino acids in a given peptide or protein. Molecular fragmentation in the gas phase may be induced by precursor

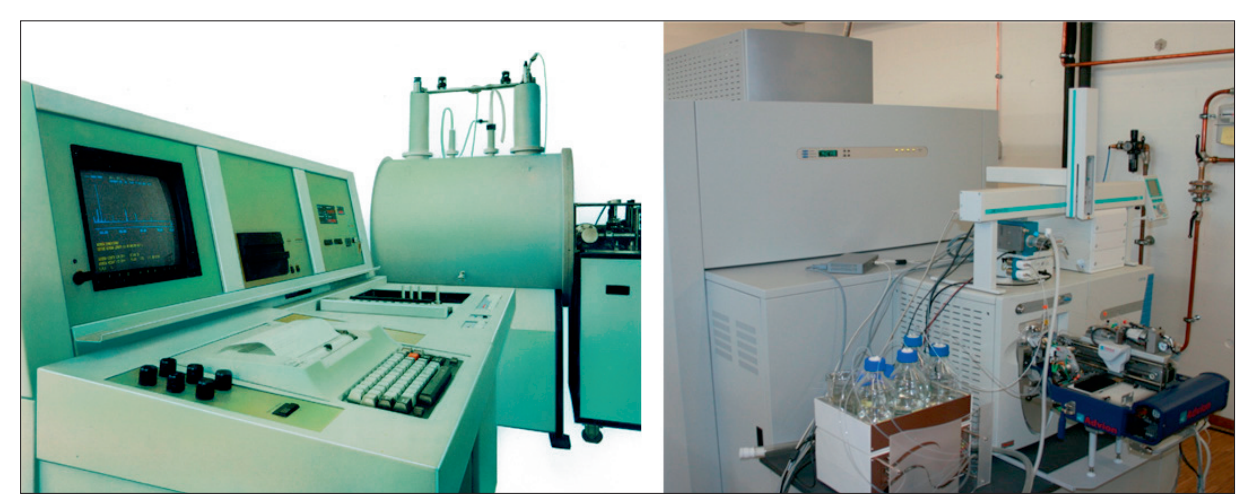

Fig. 1. Connection of the generations: Fourier transform ion cyclotron resonance mass spectrometry at ISIC, EPFL. (Left) Instrument of Prof. Dr. Tino Gäumann laboratory in the late XXth century (produced by Spectrospin AG, Switzerland, later acquired by Bruker Germany). (Right) The current state-of-the-art instrument in Prof. Dr. Yury Tsybin laboratory in the XXI century (Thermo Scientific, Bremen, Germany). ion interaction with neutral gas molecules (CID, for either positively or negatively charged precursor ions), radical anions (ETD for positively multiply charged precursor ions) or radical cations (nETD for negatively multiply charged precursor ions), photons (IRMPD, [22] UVPD, $\mathrm{EPD}^{[23]}$ for both positively and negatively charged precursor ions), and electrons $\left(E C D^{[24]}\right.$ for positively multiply charged and $\mathrm{EDD}^{[23]}$ for negatively multiply charged precursor ions). The most widely employed MS/MS method for a number of years is collision induced dissociation (CID) that cleaves the peptide bond and gives mainly $b / y$ pairs of fragments. Despite its infancy, electron transfer dissociation (ETD) is the most commonly employed complementary MS/MS method to CID and provides orthogonal information by cleaving the $\mathrm{N}-\mathrm{C}_{\alpha}$ peptide backbone bond and giving primarily $c / z$ pairs of fragments. ${ }^{[25]}$ Fig. 3 shows an example of a typical MS/MS application for peptide sequencing: IRMPD (top panel) and ECD (bottom panel) of a synthetic peptide H-CHEMISTRY-OH allow the nature and order of amino acids to be revealed in this peptide. The peptide, that we designed in recognition of the International Year of Chemistry (2011), was produced in-house by solid-state synthesis (Peptide Synthesis Facility, University of Lausanne, Switzerland) and used without further purification. It was first dissolved in pure water yielding a $1 \mathrm{mM}$ stock solution that was further diluted in an electrospray solution (water:acetonitrile (50:50 v/v) with addition of $0.1 \%$ formic acid) to a final concentration of $1-10 \mu \mathrm{M}$. The peptide was directly infused with electrospray ionization (ESI) using a nanospray ionization automated interface (Triversa nanomate, Advion Biosciences, Ithaca, NY) at a flow rate around $300 \mathrm{~nL} / \mathrm{min}$. The experiments were performed on a hybrid linear ion trap Fourier transform ion cyclotron resonance mass spectrometer (LTQ FT-ICR MS, 

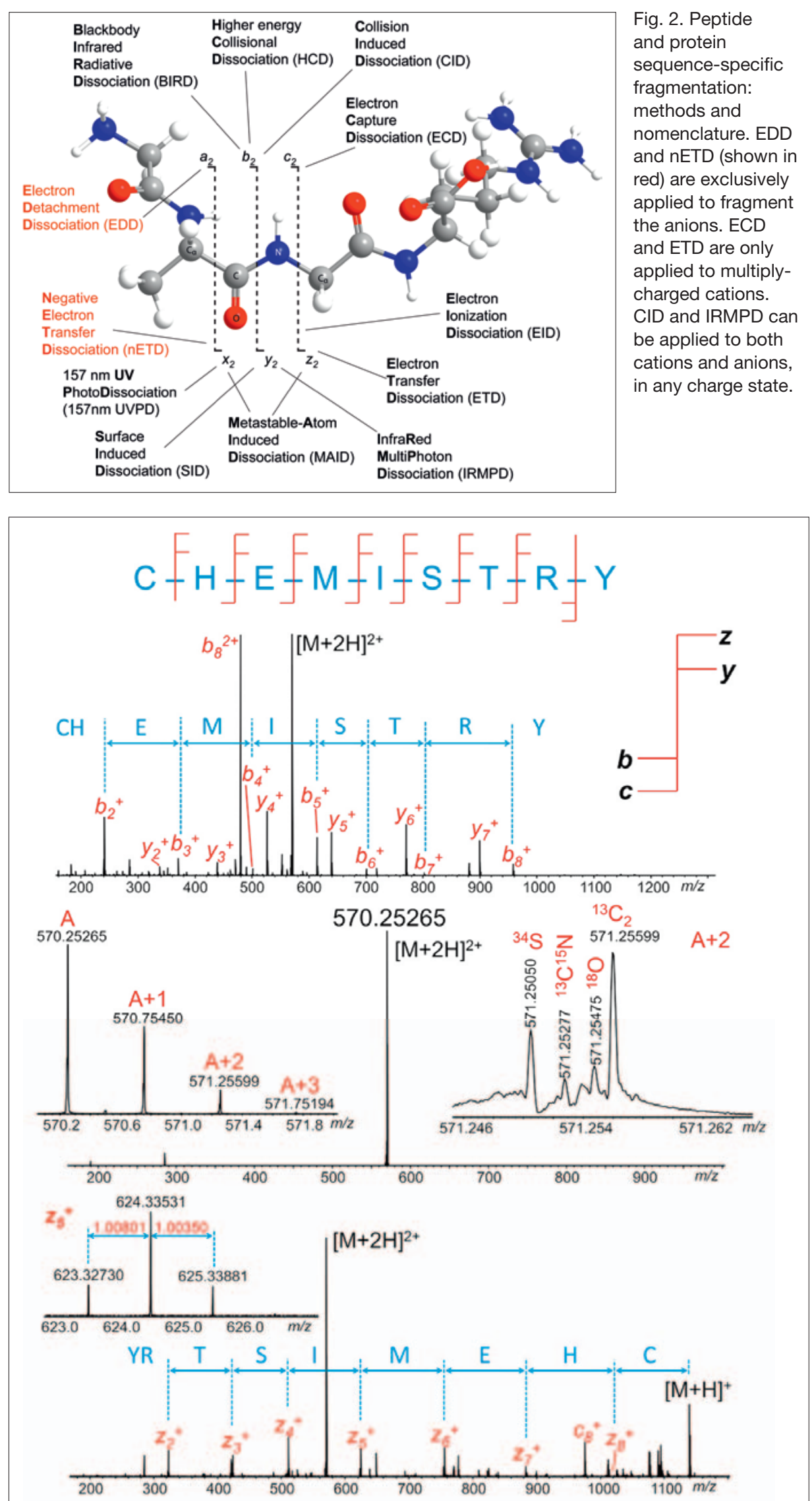

Fig. 3. High-resolution and tandem mass spectrometry provides elemental composition and primary structure information (sequence) on peptides and allows protein identification and de novo characterization. Here, doubly protonated peptide with a sequence $\mathrm{H}-\mathrm{CHEMISTRY}-\mathrm{OH}$ is fragmented with (top) absorption of photons in the IR wavelength range, IRMPD; and (bottom) capturing of low energy electrons, ECD. Insets show isotopic resolution of a doubly protonated parent (precursor) peptide (middle panel, left inset), isotopic fine structure of the peptide (middle panel, right inset) and structure of a product ion isotopic cluster indicating an overlap of an evenelectron specie and an odd-electron (radical) one (bottom panel, left inset).
Thermo Scientific, Bremen, Germany) equipped with a $10 \mathrm{~T}$ superconducting magnet at ISIC, Fig. 1, right. Doubly protonated precursor peptides were externally isolated in the linear ion trap (LTQ) using an isolation window of $4 \mathrm{~m} / \mathrm{z}$ units, prior to ion transfer to the ICR ion trap and subsequent ion manipulation following standard MS/MS procedures. Electron capture dissociation (ECD) was performed with lowenergy $(<1 \mathrm{eV})$ electrons using a pencil electron beam from a dispenser cathode located in the high magnetic field region. [26,27] The dispenser cathode was located slightly off-axis to allow a photon beam to enter the ICR ion trap at an angle for simultaneous or consecutive IR activation or dissociation experiments. The instrument is equipped with a standard $\mathrm{CO}_{2}$ laser operating at $10.6 \mu \mathrm{m}$ with $25 \mathrm{~W}$ maximum power output. An optimization of the electron injection phase is typically performed to take into account the ion cloud position. ${ }^{[28]}$ Note, in the particular case of a peptide $\mathrm{H}-\mathrm{CHEMISTRY-OH,} \mathrm{IRMPD} \mathrm{shows} \mathrm{pep-}$ tide sequencing through the $b$-ions, that is from the $\mathrm{N}$-terminus to the $\mathrm{C}$-terminus, whereas ECD reads peptide sequence through the z-ions, thus in an inverse order (Fig. 3). In general, the type of generated fragment ions is a function of charge location on a peptide. Furthermore, the product ion abundance distribution is believed to contain information on the peptide and protein secondary and, potentially, tertiary structures in the gas phase. ${ }^{[29-33]}$ The radical nature of the ion-electron interactions is manifested through the influence of amino acid radical stability properties on the product ion abundance. ${ }^{[34,35]}$

Fig. 3 also demonstrates that direct mass measurements of an intact molecule may provide different structural levels of information as a function of resolving power. Information on macromolecular, e.g. protein, charge states, adducts and even isotopes can be derived from mass spectra acquired with low- to medium resolving power $(\sim 0.001-100$ per unit of molecular weight) (Fig. 3, middle panel, left inset). The in-depth structural analysis, e.g. elemental composition from isotopic fine structure information, requires at least an order of magnitude higher resolving power per unit of molecular weight (Fig. 3, middle panel, right inset). Although the stateof-the-art high magnetic field FT-ICR MS may provide this level of performance, e.g. resolving power of a few million at $\mathrm{m} / \mathrm{z}=$ 100-2000, it requires a long experimental time, e.g. 1-20 seconds per single mass spectrum acquisition. Therefore, these mass measurements are incompatible with the current and near-future requirements for fast data acquisition, enforced by the short peptide and protein elution time from the chromatographic column. Possible so- 
lutions to this problem could be in both hardware and signal processing development, vide infra.

The recently achieved progress in the diverse MS application areas clearly demonstrates the limitations of the current state-of-the-art and the need for further improvement of its analytical characteristics to address the increasingly growing complexity of the materials of biological and chemical nature and their interaction pathways. ${ }^{36-38]}$ The result of the recent investments in the MS field, especially in the life science and clinical directions, is primarily an intellectual and technological progress, whereas substantial economical yields are still awaited and highly anticipated. Similarly, the sustainability of energy and fuel production and logistics is highly sensitive to the molecular content. Specifically, an important environmental and economical problem of heteroatoms, e.g. sulfur, accurate speciation becomes increasingly important with the current shift from light to heavy crude oil production. ${ }^{39]}$ However, the required level of analytical performance of the measurement systems is not readily provided today. Therefore, the current stage of MS applications confirms that investing into basic chemical and physical research, as well as improving the sample preparation and formulation through promotion of the comprehensive dialogue of the mass spectrometrists with the life science researchers, clinicians and environmental scientists, are as essential as ever to be prepared for the growing application challenges. Selected approaches aimed to improve the performance of the current MS technology are briefly discussed below.

\section{Solution-phase Chemistry}

Higher throughput, more reproducible, repeatable, robust and efficient methods of sample preparation, including sample fractionation and purification, are required. For example, the multiple-enzyme strategies leading to MS/MS-like fragmentation in solution and middle down proteomics (enzymatic digestion to 20-50 amino acid long peptides, instead of the currently produced 10-15 amino acid long peptides) are underway. Furthermore, comprehensive description of peptide and protein separation by liquid chromatography will allow retention time information to be added as a complementary and orthogonal source of data to the mass measurements. Within the on-going collaboration between our laboratory and Dr. Gorshkov's group (Russian Academy of Sciences, Moscow, Russia), a comprehensive model of chromatographic separation of proteins based on their behavior near the critical conditions (between the size exclusion and liquid adsorption modes of separation) is under development.

\section{Gas-phase Chemistry}

The advances here are envisioned in molecular conformer separation with ion mobility and subsequent high efficiency MS/ MS method and technique development. Already today, a resolving power of 80-100 can be achieved on commercial ion mobility mass spectrometers at the sensitivity level sufficient for the entry-level life-science applications. ${ }^{[40,41]}$ Importantly, one of the world's leading manufacturing companies in this field is Tofwerk AG, located in Thun, Switzerland. Similarly to the retention time information, ion separation by their threedimensional structures in the gas phase provides a complementary and orthogonal source of data to the mass measurements. Development of novel and improved methods of (bio)molecular fragmentation is progressing through the implementation of novel interacting agents, e.g. photons in the unexplored wavelength regions and rationally designed molecules (neutral, charged and radicals) with specific chemical properties. Hybrid MS/MS methods, e.g. activated ion ECD and ETD, also have been shown to provide a significant improvement in the analytical applications. ${ }^{[42]}$

\section{Instrument Development}

FT-ICR MS hardware development follows the implementation of ultra-high, e.g. $21 \mathrm{~T}$, magnetic field environments together with high acquisition speed and harmonized ICR ion traps. ${ }^{[43,44]}$ The closest performance mass spectrometer to the high field FT-ICR MS is the recently introduced Orbitrap FTMS. ${ }^{[45]}$ This is an electrostatic mass analyzer that demonstrates superior performance in terms of overall instrument sensitivity to the FT-ICR MS, primarily due to higher ion transmission efficiency, but does not yet allow the same level of resolving power to be reached routinely. However, recent progress in Orbitrap FTMS development has significantly reduced the gap between Orbitrap and ICR FTMS in terms of obtained resolving power required for the mainstream MS applications, 60-200 k. ${ }^{[46]}$ Finally, the state-of-the-art time-of-flight (TOF) mass analyzers have demonstrated a substantial progress in the recent years and now offer $30-60 \mathrm{k}$ resolving powers achieved at comparable times to the high field ICR and Orbitrap FTMS. ${ }^{[47]}$ The most recent development in TOF MS technology aimed to increase the resolving power is related to the implementation of the FTmethods applied to the induced-current generated ion signals. We envision that within the next five years we will witness a serious breakthrough in this technology, which may become commercial.

\section{Data Processing}

Incredible progress in computational power and high frequency electronics will drive the MS-based technology performance to the new level. Signal processing development, which appears to be more realistic and less expensive than hardware improvement, has received particular attention in recent years. A number of groups, including ours, are in the process of tailoring super-resolution signal processing methods, e.g. the filter diagonalization method (FDM), to the needs of the ICR and Orbitrap MS.[48] The goal is to replace FT-based signal processing with methods that would require 10-100 times shorter transient time-domain signals to yield a similar level of resolving power. In the distant future this may allow the next important step in resolving power increase to be made that could enable the differentiation between the isomers of a given molecule, which is not achievable today. ${ }^{[15]}$ In other words, we would be able to weigh a chemical bond in organic molecules to confirm their structure directly from the MS data! Indeed, the resolving power required to differentiate the two isomers is (estimation with calculated heats of formation):

$$
R=\frac{M}{\Delta m}=10^{11} \frac{M[\mathrm{Da}]}{\Delta E[\mathrm{~kJ} / \mathrm{mol}]}
$$

giving the approximate resolving power for differentiation between benzene and bicyclopropenyl, both with the elemental composition $\mathrm{C}_{6} \mathrm{H}_{6}$ and the difference in mass $\Delta m=5 \mathrm{nDa}$,

$$
\Delta m=5 \mathrm{nDa}, R=\frac{M}{\Delta m} \approx 10^{11} \frac{78}{500} \approx 10^{10} .
$$

To conclude, high-resolution and tandem mass spectrometry have already revolutionized the way we treat sustainability, energy and health problems today. However, incremental progress in highresolution mass spectrometry performance and tandem mass spectrometry efficiency is needed and highly anticipated. The particular application fields that capitalize upon high-resolution mass measurements and efficient tandem mass spectrometry are petroleomics, ${ }^{[49]}$ metabolomics, bottom-up and top-down proteomics, ${ }^{[11,50]}$ and mass spectrometry-based imaging. Furthermore, a truly interdisciplinary approach is needed to move MS into the clinics. Researchers from all the complementary areas, for example, mass spectrometry, NMR and pathology, thus need to open their data to the community. However, in addition to the intellectual properties and confidential patient information, the quality of the data is a major concern. The noise in information available is still too 
high to integrate it into the interdisciplinary approach. The avenues of method and technique development in high resolution and tandem mass spectrometry described above, currently under investigation at the ISIC's Biomolecular mass spectrometry laboratory, should significantly contribute to the improved data quality and lead to advances in the corresponding applications.

\section{Acknowledgements}

We are grateful for financial support from EPFL, the Swiss National Science Foundation (SNF project 200021-125147/1), the Joint Research Project of Scientific \& Technological Cooperation Program Switzerland-Russia (grant agreement 128357), and the SATW FranceSwitzerland cross-border mobility project.

Received: July 13, 2011

[1] R. Aebersold, M. Mann, Nature 2003, 422, 198

[2] A. Dove, Science 2010, 328, 920

[3] K. Dettmer, P. A. Aronov, B. D. Hammock, Mass Spectrom. Rev. 2007, 26, 51.

[4] L. A. McDonnell, R. M. A. Heeren, Mass Spectrom. Rev. 2007, 26, 606.

[5] L. M. F. de Godoy, J. V. Olsen, J. Cox, M. L. Nielsen, N. C. Hubner, F. Frohlich, T. C. Walther, M. Mann, Nature 2008, 455, 1251.

[6] B. F. Cravatt, G. M. Simon, J. R. Yates, Nature 2007, 450, 991.

[7] J. B. Fenn, M. Mann, C. K. Meng, S. F. Wong, C. M. Whitehouse, Science 1989, 246, 64.

[8] F. Hillenkamp, M. Karas, R. C. Beavis, B. T. Chait, Anal. Chem. 1991, 63, A1193.

[9] A. Wolf-Yadlin, S. Hautaniemi, D. A. Lauffenburger, F. M. White, Proc. Nat. Acad. Sci. USA 2007, 104, 5860

[10] R. M. Ewing, P. Chu, F. Elisma, H. Li, P. Taylor, S. Climie, L. McBroom-Cerajewski, M. D. Robinson, L. O'Connor, M. Li, R. Taylor, M. Dharsee, Y. Ho, A. Heilbut, L. Moore, S Zhang, O. Ornatsky, Y. V. Bukhman, M. Ethier, Y. Sheng, J. Vasilescu, M. Abu-Farha, J. P. Lambert, H. S. Duewel, Stewart, II, B. Kuehl, K. Hogue, K. Colwill, K. Gladwish, B. Muskat, R. Kinach, S. L. Adams, M. F. Moran, G. B. Morin, T. Topaloglou, D. Figeys, Mol. Sys. Biol. 2007, 3, 89 .
[11] N. L. Kelleher, Anal. Chem. 2004, 76, 196A

[12] J. Cox, M. Mann, Cell 2007, 130, 395.

[13] N. L. Kelleher, H. Y. Lin, G. A. Valaskovic, D. J. Aaserud, E. K. Fridriksson, F. W. McLafferty, J. Am. Chem. Soc. 1999, 121, 806.

[14] A. G. Marshall, C. L. Hendrickson, Ann. Rev. Anal. Chem. 2008, 1, 579.

[15] A. G. Marshall, C. L. Hendrickson, S. D. H. Shi, Anal. Chem. 2002, 74, 252 A.

[16] A. G. Marshall, R. P. Rodgers, Proc. Nat. Acad. Sci. USA 2008, 105, 18090.

[17] I. V. Chernushevich, A. V. Loboda, B. A. Thomson, J. Mass Spectrom. 2001, 36, 849.

[18] A. G. Marshall, C. L. Hendrickson, Int. J. Mass Spectrom. 2002, 215, 59.

[19] T. M. Schaub, C. L. Hendrickson, S. Horning, J. P. Quinn, M. W. Senko, A. G. Marshall, Anal. Chem. 2008, 80, 3985.

[20] P. Pfandler, G. Bodenhausen, J. Rapin, M. E. Walser, T. Gaumann, J. Am. Chem. Soc. 1988, $110,5625$.

[21] Y. O. Tsybin, M. Ramstrom, M. Witt, G. Baykut, P. Hakansson, J. Mass Spectrom. 2004, 39, 719.

[22] D. P. Little, J. P. Speir, M. W. Senko, P. B. Oconnor, F. W. McLafferty, Anal. Chem. 1994, 66, 2809.

[23] V. Larraillet, A. Vorobyev, C. Brunet, J. Lemoine, Y. O. Tsybin, R. Antoine, P. Dugourd, $J$. Am. Soc. Mass Spectrom. 2010, 21, 670.

[24] R. A. Zubarev, N. L. Kelleher, F. W. McLafferty, J. Am. Chem. Soc. 1998, 120, 3265.

[25] R. A. Zubarev, A. R. Zubarev, M. M. Savitski, J. Am. Soc. Mass Spectrom. 2008, 19, 753.

[26] Y. O. Tsybin, P. Hakansson, B. A. Budnik, K. F. Haselmann, F. Kjeldsen, M. Gorshkov, R. A. Zubarev, Rapid Comm. Mass Spectrom. 2001. 15,1849

[27] Y. O. Tsybin, J. P. Quinn, O. Y. Tsybin, C. L. Hendrickson, A. G. Marshall, J. Am. Soc. Mass Spectrom. 2008, 19, 762.

[28] Y. O. Tsybin, C. L. Hendrickson, S. C. Beu, A G. Marshall, Int. J. Mass Spectrom. 2006, 255 , 144.

[29] H. Ben Hamidane, H. He, O. Y. Tsybin, M. R. Emmett, C. L. Hendrickson, A. G. Marshall, Y. O. Tsybin, J. Am. Soc. Mass Spectrom. 2009, 20, 1182 .

[30] B. A. Budnik, M. L. Nielsen, J. V. Olsen, K. F. Haselmann, P. Horth, W. Haehnel, R. A. Zubarev, Int. J. Mass Spectrom. 2002, 219, 283.

[31] K. Breuker, H. B. Oh, C. Lin, B. K. Carpenter, F. W. McLafferty, Proc. Nat. Acad. Sci. USA 2004, 101, 14011
[32] M. M. Savitski, F. Kjeldsen, M. L. Nielsen, S. O. Garbuzynskiy, O. V. Galzitskaya, A. K. Surin, R. A. Zubarev, Angew. Chem. Int. Ed. 2007, 46, 1481.

[33] Z. Zhang, J. Bordas-Nagy, J. Am. Soc. Mass Spectrom. 2006, 17, 786 .

[34] A. Vorobyev, H. B. Hamidane, Y. O. Tsybin, $J$. Am. Soc. Mass Spectrom. 2009, 20, 2273.

[35] H. Ben Hamidane, A. Vorobyev, M. Larregola, A. Lukaszuk, D. Tourwe, S. Lavielle, P. Karoyan, Y. O. Tsybin, Chem. Eur. J. 2010, 16, 4612.

[36] M. Mann, Nat. Meth. 2009, 6, 717.

[37] R. Aebersold, Nat. Meth. 2009, 6, 411.

[38] A. W. Bell, E. W. Deutsch, C. E. Au, R. E. Kearney, R. Beavis, S. Sechi, T. Nilsson, J. J. M. Bergeron, Nat. Meth. 2009, 6, 423.

[39] J. M. Purcell, P. Juyal, D. G. Kim, R. P. Rodgers, C. L. Hendrickson, A. G. Marshall, Energy \& Fuels 2007, 21, 2869.

[40] A. B. Kanu, P. Dwivedi, M. Tam, L. Matz, H. H. Hill, J. Mass Spectrom. 2008, 43, 1 .

[41] B. T. Ruotolo, J. L. P. Benesch, A. M Sandercock, S. J. Hyung, C. V. Robinson, Nature Protocols 2008, 3, 1139.

[42] Y. O. Tsybin, H. He, M. R. Emmett, C. L. Hendrickson, A. G. Marshall, Anal. Chem. 2007, 79, 7596.

[43] E. Nikolaev, I. Boldin, R. Jertz, G. Baykut, J. Am. Soc. Mass Spectrom. 2011, 22, 1125.

[44] A. Tolmachev, E. Robinson, S. Wu, R. Smith, L. Pasa-Tolic, J. Am. Soc. Mass Spectrom. 2011. 22,1 .

[45] A. Makarov, E. Denisov, O. Lange, J. Am. Soc. Mass Spectrom. 2009, 20, 1391.

[46] J. V. Olsen, M. L. Nielsen, N. E. Damoc, J. Griep-Raming, T. Moehring, A. Makarov, J. Schwartz, S. Horning, M. Mann, Mol. Cell. Proteomics 2009, S40.

[47] R. G. Hartmer, D. A. Kaplan, C. Stoermer, M. Lubeck, M. A. Park, Rapid Comm. Mass Spectrom. 2009, 23, 2273.

[48] K. Aizikov, P. B. O'Connor, J. Am. Soc. Mass Spectrom. 2006, 17, 836.

[49] A. G. Marshall, G. T. Blakney, S. C. Beu, C. L. Hendrickson, A. M. McKenna, J. M. Purcell, R. P. Rodgers, F. Xian, Eur. J. Mass Spectrom. 2010, 16, 367.

[50] B. A. Garcia, J. Am. Soc. Mass Spectrom. 2010 $21,193$. 